\title{
NLTE strontium abundances in extremely metal poor halo stars
}

\section{S.M. Andrievsky ${ }^{* \dagger}$}

Astronomical Observatory of Odessa National University, Ukraine GEPI, Observatoire de Paris-Meudon, F-92125 Meudon Cedex, France

E-mail: scan@deneb1.odessa.ua

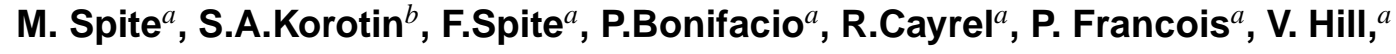

GEPI, Observatoire de Paris-Meudon, F-92125 Meudon Cedex, France

${ }^{b}$ Astronomical Observatory of Odessa National University, Ukraine

E-mail: Monique.Spite@obspm.fr, serkoreskyline.od.ua

\begin{abstract}
NLTE analysis of the Sr II lines in the spectra of 35 extremely metal poor halo stars has been performed. For this we have created Sr II atomic model consisting of 44 levels of Sr II with $\mathrm{n}<13$ and $1<6$, as well as the ground level of Sr III. The following lines of Sr II have been used to derive the strontium abundance in our program stars: resonance lines $4077 \AA$, $4215 \AA$, and subordinate lines $10036 \AA, 10327 \AA, 10914 \AA$. Strontium abundance has been analyzed together with previously determined NLTE barium abundance in these stars. We show that within the interval of metallicities $([\mathrm{Fe} / \mathrm{H}]$ is from -2.2 to -4.2$)$ the ratio $[\mathrm{Sr} / \mathrm{Ba}]$ is almost independent of $[\mathrm{Fe} / \mathrm{H}]$. The mean value is around zero with scatter from about -0.5 to 0.5 . One star of our sample show significant deviation from the bulk of stars. Its $[\mathrm{Sr} / \mathrm{Ba}]$ ratio is 1.4 . Obtained results are discussed from the evolutionary point of view.
\end{abstract}

11th Symposium on Nuclei in the Cosmos

19-23 July 2010

Heidelberg, Germany.

\footnotetext{
* Speaker.

$\dagger$ SMA and SAK would like to thank administration of Paris-Meudon Observatory and SCOPES grant No. IZ73Z0128180/1 that supported the work and presentation.
} 


\section{Introduction}

A few years ago we started a program of the NLTE spectroscopic analysis of extremely metalpoor (EMP) stars. As a consequence, to improve the determination of the abundance of $\mathrm{Sr}$ and $\mathrm{Ba}$ at low metallicity, we decided to reanalyse the sample of the EMP stars previously studied by Francois et al. [1] and Bonifacio et al. [2] in the frame of the ESO Large Program "First stars, first nucleosynthesis". In these stars we have determined the abundance of Sr based on NLTE computations of the line profiles. These abundances are combined with the NLTE computations of $\mathrm{Ba}$ (see Andrievsky et al., [3]) to study the evolution of the $\mathrm{Sr} / \mathrm{Ba}$ ratio in the early times of the Galaxy.

\section{NLTE calculations}

The Sr atomic model consists of 44 levels of Sr II with $\mathrm{n}<13$ and $1<6$, as well as the ground level of Sr III.

The NLTE strontium abundance was determined with the help of modified MULTI code (Carlsson [4]). Modifications are described in Korotin et al. [5].

Since the resonance Sr II lines are blended to some extent, before comparing the NLTE strontium line profiles with observed spectrum one needs to use a combination of the NLTE and LTE synthetic spectrum. This was done through the updated LTE code SYNTHV (Tsymbal, [6]). Line parameters are given in Table 1 .

\begin{tabular}{cccc}
\hline$\lambda, \AA$ & $f$ & $\log \gamma_{\mathrm{rad}}$ & $\log \gamma_{\mathrm{VW}}$ \\
\hline 4077.7090 & $7.063 \mathrm{e}-1$ & 8.130 & -7.70 \\
4161.7920 & $1.945 \mathrm{e}-1$ & 8.110 & -7.60 \\
4215.5190 & $3.303 \mathrm{e}-1$ & 8.100 & -7.70 \\
4305.4430 & $1.811 \mathrm{e}-1$ & 8.080 & -7.60 \\
10036.6530 & $1.219 \mathrm{e}-2$ & 7.340 & -7.63 \\
10327.3110 & $7.393 \mathrm{e}-2$ & 7.320 & -7.63 \\
10914.8870 & $5.754 \mathrm{e}-2$ & 7.270 & -7.63 \\
\hline
\end{tabular}

Table 1: Parameters of the Sr II lines.

As a test we calculated profiles for the six Sr II lines in the solar spectrum (visual and IR range, Kurucz's et al. [7] solar flux spectrum) using the Kurucz's [8] solar atmosphere model.

Fig. 1 shows that the Sr II lines in solar spectrum and HD 122563 that belong to the different multiplets are well reproduced with one strontium abundance.

In Fig. 2 we show the dependence of NLTE-LTE abundance correction upon effective temperature and other parameters.

Fig. 3 (left plot) shows the increase of the scatter with decreasing metallicity. Figure 3 (right plot) shows the evolution of $\mathrm{Sr} / \mathrm{Fe}$ vs. $\mathrm{Ba} / \mathrm{Fe}$ at low metallicity. Within the simplest scenario of chemical evolution, it is supposed that in the early phases of this evolution, the iron abundance is low and the stars of the AGB (asymptotic giant branch) have not yet produced neutron capture 

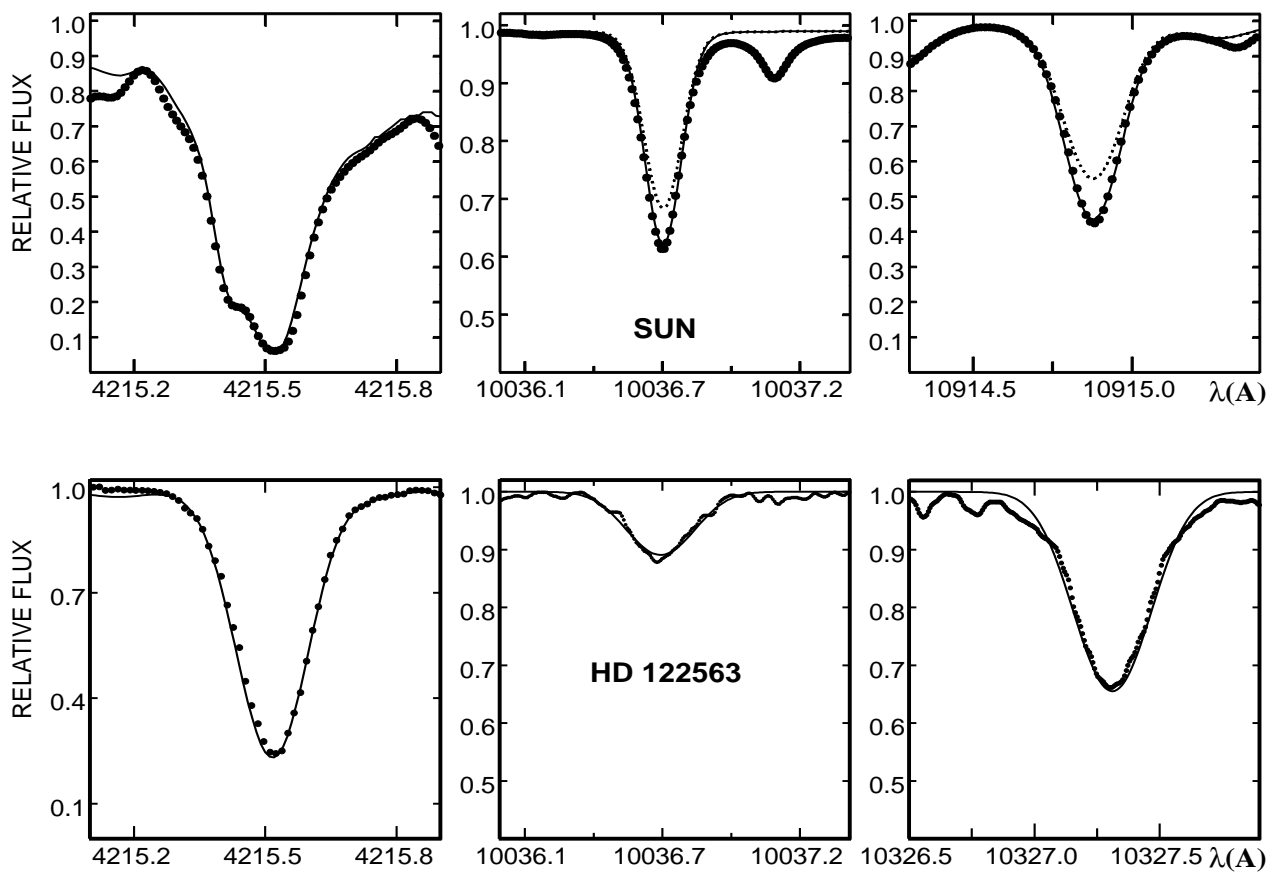

Figure 1: Profile fitting for the Sr II lines in the solar spectrum and HD 122563 spectrum. Observed spectrum - dots, NLTE profiles - continuous line, LTE profiles - dotted line.
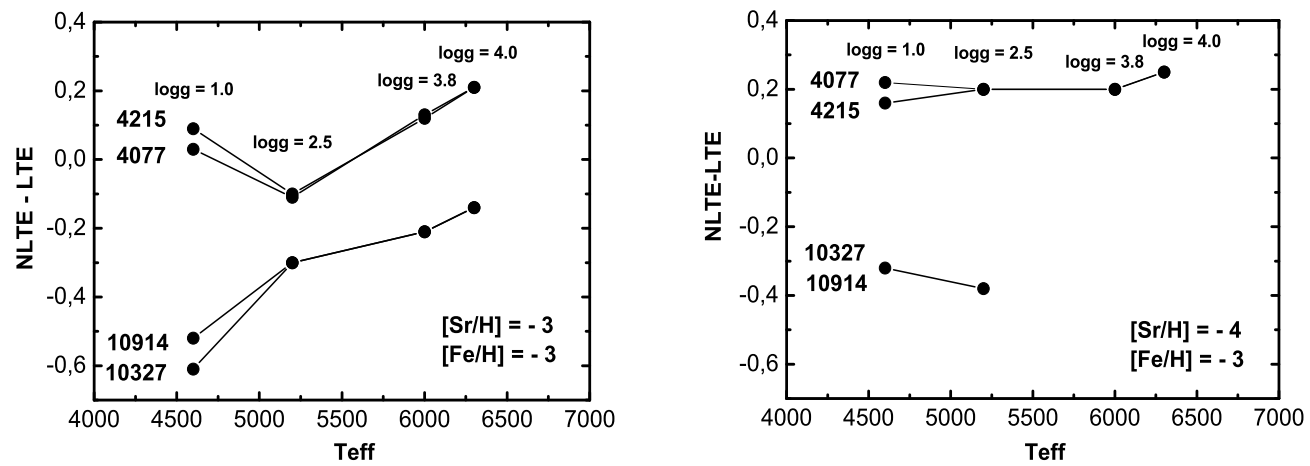

Figure 2: NLTE correction as a function of $\mathrm{T}_{\mathrm{eff}}$ and $\log g$ for the different metallicities.

elements by the s-process. The production of $\mathrm{Sr}$ (and $\mathrm{Ba}$ ) at extremely low metallicities would therefore be due only to an early r-process which takes place, for example,in fast evolving SNe II.

Fig. 4 (left plot) shows the comparison of $[\mathrm{Sr} / \mathrm{Ba}]$ vs. $[\mathrm{Fe} / \mathrm{H}]$ for our program stars together with the plot of the theoretical LEPP model predictions (Travaglio et al. [10]). The corresponding theoretical curve is not far from the upper envelope of the data points, and a varied amount of such a LEPP process (e.g. by inhomogeneous mixing) could explain the distribution of the stars in the figure. Fig. 4 (right plot) shows the amplitude of the scatter of the ratio $\mathrm{Sr} / \mathrm{Ba}$ : the amplitude varies 

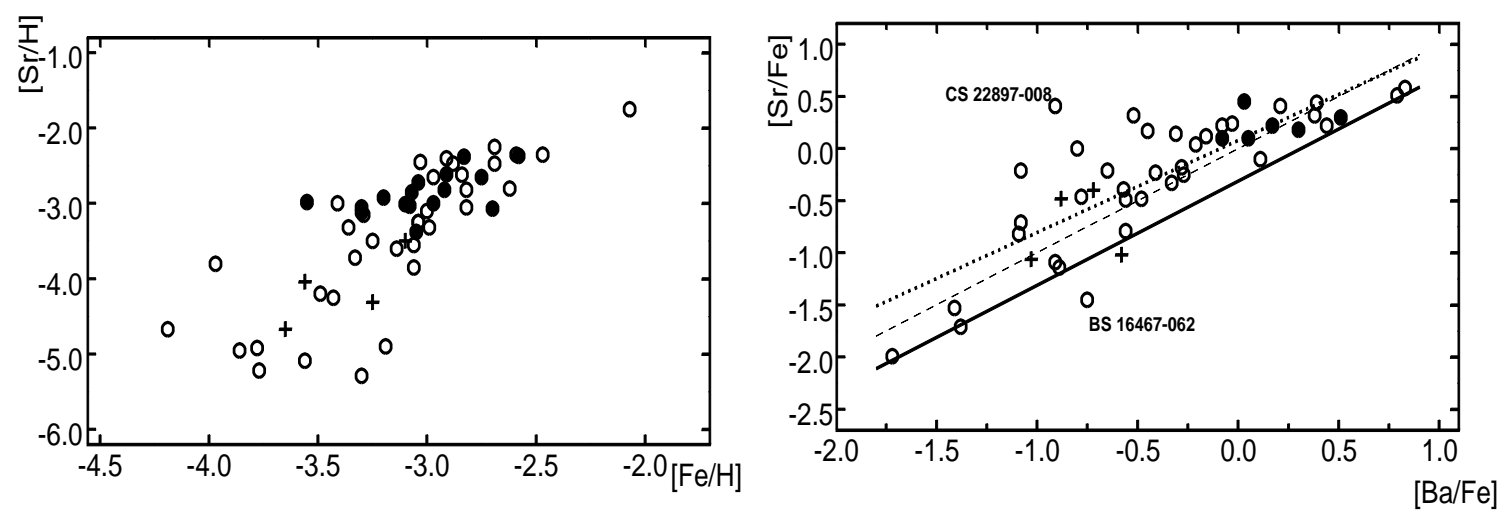

Figure 3: Left: $[\mathrm{Sr} / \mathrm{H}]$ vs. $[\mathrm{Fe} / \mathrm{H}]$ in our program stars: dwarfs - closed circles, giants - open circles, new added giants - plus signs. Right: [Sr/Fe] vs. [Ba/Fe]. Wasserburg \& Qian [9] the three-component model (three sources of $\mathrm{Sr}$ and Ba production: HyperNe, the normal-mass $\mathrm{SNe}$ and low-mass $\mathrm{SNe}$ ) prediction with $[\mathrm{Sr} / \mathrm{Ba}]_{H}=-0.31-$ continuous line, the same with $[\mathrm{Sr} / \mathrm{Ba}]_{H}=0$ - dashed line, linear regression - dotted line.
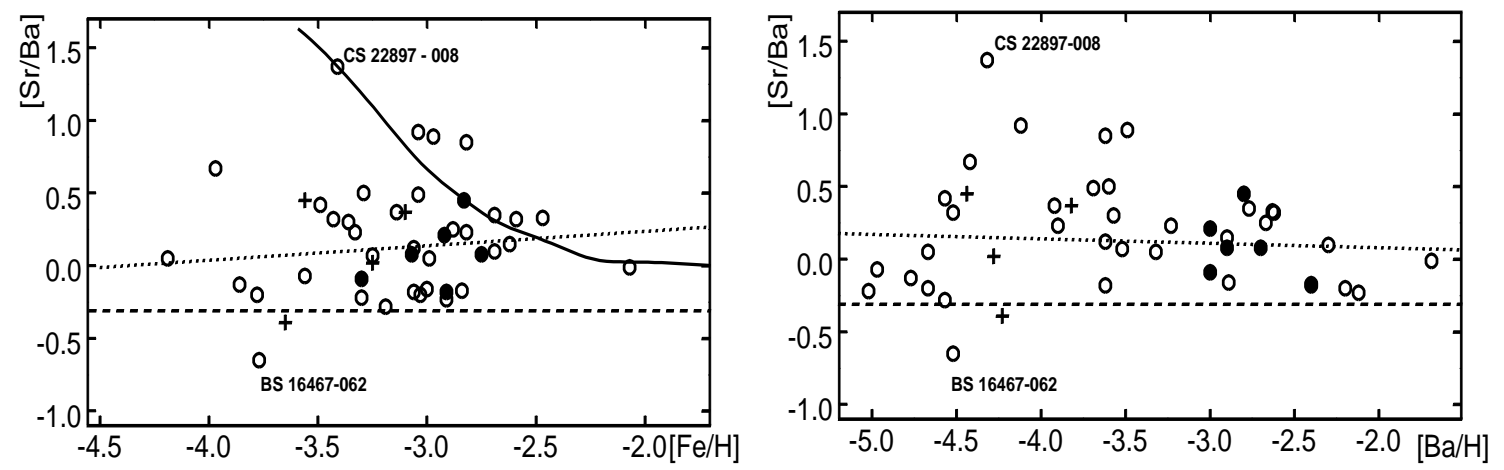

Figure 4: Left: $[\mathrm{Sr} / \mathrm{Ba}]$ vs. [Fe/H]. LEPP model prediction (Travaglio et al. [10]) - continuous line, $[\mathrm{Sr} / \mathrm{Ba}]_{H}$ $=-0.31-$ dashed line, linear regression - dotted line. Right: $[\mathrm{Sr} / \mathrm{Ba}]$ vs. $[\mathrm{Ba} / \mathrm{H}] .[\mathrm{Sr} / \mathrm{Ba}]_{H}=-0.31-$ dashed line, linear regression - dotted line.

strongly along with the value of the Ba abundance.

Fig. 5 (left plot) presents a pattern rather similar to the one found in Fig. 3, but here the iron content is indicated by the sizes of the circles: the different steps of iron-production in the Galaxy are then identified. Again, the lower envelope reflects the classical r-process, whatever the metallicity. The largest deviations from the r-process is in the range $-4.3<[\mathrm{Ba} / \mathrm{H}]<-$ 3.3, corresponding to "medium" or "large" iron abundances. Figure 5 (right plot) shows again a simultaneous enrichment of $\mathrm{Sr}$ and $\mathrm{Ba}$, but with an indication of iron abundance .

\section{Conclusion}

Summarizing, the three-component model ( $\mathrm{HNe}$, the normal-mass $\mathrm{SNe}$ and low-mass $\mathrm{SNe}$ ) with inhomogeneous distribution of synthesized $\mathrm{Fe}, \mathrm{Sr}$ and $\mathrm{Ba}$ nuclei seems to be able to explain observed Sr-Ba relations in EMP stars. We did not discuss observational results on strontium and 

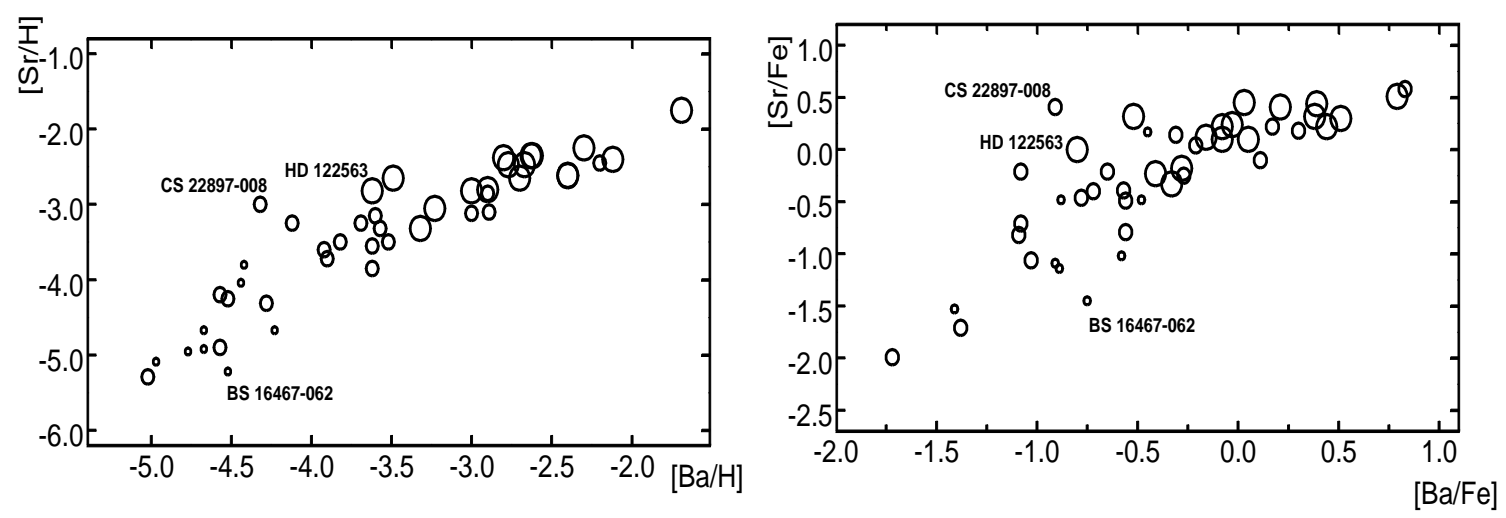

Figure 5: Left: $[\mathrm{Sr} / \mathrm{H}]$ vs. $[\mathrm{Ba} / \mathrm{H}]$. Stars with $[\mathrm{Fe} / \mathrm{H}]<-3.5-$ small circles, $-3.5<[\mathrm{Fe} / \mathrm{H}]<-3.0-$ intermediate circles, $[\mathrm{Fe} / \mathrm{H}]>-3.0$ - large circles. Right: $[\mathrm{Sr} / \mathrm{Fe}]$ vs. $[\mathrm{Ba} / \mathrm{Fe}]$.

barium abundances reported in the literature, since all of them are based on the LTE analysis. Our conclusion does not reject the possibility of the LEPP existence. Presented results merely do not provide a firm ground to believe that this process exist in the nature. We think that the most reliable means to check the LEPP hypothesis would be the complete NLTE re-analysis of all available literature data on $\mathrm{Sr}$ and $\mathrm{Ba}$ abundance in the very- and extremely metal-poor stars.

\section{References}

[1] P. Francois, E. Depagne, V. Hill et al., $A \& A$ (2007) 476, 935

[2] P. Bonifacio, M. Spite, R.Cayrel, et al., $A \& A$ (2009) 501, 519

[3] S.M. Andrievsky, M. Spite, S.A. Korotin et al., A\&A (2009) 494, 1083

[4] M. Carlsson, Uppsala Obs. Rep.(1986) 33

[5] S.A. Korotin, S.M. Andrievsky, R.E. Luck, A\&A (1999) 351, 168

[6] V.V. Tsymbal, Model Atmosph. \& Spectrum Synthesis, ASP Conf. Ser. (1996), 108

[7] R.L. Kurucz, I. Furenlid, J. Brault, L. Testerman, Solar flux atlas from 296 to $1300 \mathrm{~nm}$ (1984)

[8] R.L. Kurucz, ASP Conference Series (1996) 108

[9] G.J. Wasserburg, Y.-Z. Qian, PASA (2009) 26, 184

[10] C. Travaglio, R. Gallino, E. Arnone, et al., ApJ (2004) 601, 864 